\title{
Dermatitis herpetiforme como manifestación extraintestinal gluten inducida en la enfermedad celíaca: estudio retrospectivo de pacientes que se asisten en el Hospital de Clínicas Dr. Manuel Quintela
}

Dermatitis herpetiformis as a gluten induced extraintestinal manifestation in coeliac disease: retrospective study of patients assisted at "Dr. Manuel Quintela" University Hospital

Dermatite herpetiforme como manifestação extraintestinal induzida por glúten na doença celíaca: estudo retrospectivo de pacientes atendidos no Hospital de Clínicas "Dr. Manuel Quintela"

Mariana Lizarraga', Pilar Echenagusia², Julio Magliano³,

Carlos Bazzano ${ }^{4}$, Miguel Martínez Asuaga ${ }^{5}$

\section{Resumen}

Introducción: la dermatitis herpetiforme (DH) es una enfermedad cutánea inflamatoria que cursa en empujes y remisiones. Es una manifestación extraintestinal de la enfermedad celíaca (EC), de la que puede ser la primera manifestación clínica(1). La enfermedad celíaca es una enfermedad sistémica autoinmune, asociada con enteropatía, desencadenada por la ingesta de gluten en individuos genéticamente predispuestos ${ }^{(2-4)}$. Se ha demostrado una asociación con el HLA DQ2 y HLA DQ8 en ambas enfermedades ${ }^{(5)}$.

Objetivo: mostrar las características de los pacientes con DH y EC que se asisten en las policlínicas de dermatología y gastroenterología del Hospital Universitario Dr. Manuel Quintela.

Metodología: se realizó un estudio descriptivo, observacional, retrospectivo y longitudinal de una serie de casos. Criterios de inclusión: pacientes asistidos en servicios de dermatología y gastroenterología del Hospital de Clínicas, con diagnóstico de DH confirmado con histopatología y/o inmunofluorescencia directa; desde julio de 2000 a junio de 2018 inclusive. Quedaron excluidos aquellos pacientes en los que no se pudo acceder al estudio histopatológico de piel.

Resultados: incluimos un total de 15 pacientes, 9 de sexo masculino y 6 de sexo femenino. La edad media al diagnóstico de DH fue de 49 años. 4 pacientes recibieron tratamiento con dapsona, con rápida respuesta de la dermatosis, sin efectos adversos graves. En 13 pacientes la DH fue el síntoma guía para el diagnóstico de EC. 5 pacientes con EC asintomática, 6 con síntomas clásicos, 4 con síntomas no clásicos.

\footnotetext{
1. Dermatóloga.

2. Residente de Dermatología,

3. Profesor Adjunto de la Cátedra de Dermatología.

4. Profesor de la Cátedra de Dermatología.

5. Profesor Agregado de la Cátedra de Dermatología.

Hospital de Clínicas Dr. Manuel Quintela. Facultad de Medicina. Universidad de la República. Montevideo, Uruguay.

Correspondencia: Dr. Julio Magliano. Hospital de Clínicas. Av. Italia s/n. Montevideo, Uruguay. Correo electrónico: juliomagliano@gmail.com

Los autores no tienen conflictos de interés.

El artículo fue aprobado por el Comité de Ética del Hospital de Clínicas

Recibido: 28/6/2021

Aprobado: 18/10/2021

Attribution-NonCommercial 4.0 International (CC BY-NC 4.0)
} 


\section{Palabras clave: Dermatitis herpetiforme Enfermedad celíaca \\ Key words: Dermatitis herpetiformis Celiac disease}

\section{Introducción}

La dermatitis herpetiforme (DH) es una enfermedad cutánea inflamatoria que cursa en empujes y remisiones, caracterizada por pápulas y vesículas pruriginosas localizadas principalmente en áreas extensoras de codos, rodillas, región proximal de antebrazos y glúteos; que presentan una histopatología e inmunofluorescencia características ${ }^{(6-9)}$. Es una manifestación extraintestinal de la enfermedad celíaca (EC), de la cual puede ser la primera manifestación clínica ${ }^{(1)}$.

La enfermedad celíaca es una enfermedad sistémica autoinmune, asociada con enteropatía, desencadenada por la ingesta de gluten en individuos genéticamente predispuestos. Afecta a ambos sexos y puede desarrollarse a cualquier edad ${ }^{(2-4)}$.

Se ha demostrado una asociación con el HLA DQ2 y HLA DQ8 en ambas enfermedades ${ }^{(5,10)}$. La DH presenta una incidencia entre 11,2 a 75,3 cada 100.000 habitantes en Europa ${ }^{(11)}$. La EC es una patología frecuente. Estudios realizados en la población general en Europa, Estados Unidos y diferentes países de América Latina han encontrado una prevalencia que oscila entre 1/100-1/300 individuos, con una relación mujer hombre $2-3 / 1^{(12,13)}$. Se estima que por cada caso diagnosticado existen entre 5-10 personas no diagnosticadas ${ }^{(12)}$.

Se destaca que si bien la incidencia anual de la enfermedad celíaca ha aumentado, lo contrario ha sucedido con la dermatitis herpetiforme, ya que su incidencia anual ha descendido significativamente ${ }^{(2)}$.

El objetivo general es conocer las características sociodemográficas y clínicas de los pacientes con $\mathrm{DH}$ que se asisten en los servicios de dermatología y gastroenterología en el Hospital de Clínicas Dr. Manuel Quintela, centro de referencia nacional.

Los objetivos específicos incluyeron:

1) Caracterizar la presentación de la dermatosis en la población estudiada.

2) Caracterizar los hallazgos en la histopatología e inmunofluorescencia directa de las biopsias cutáneas.

3) Determinar la presencia de síntomas digestivos, extradigestivos, atrofia vellositaria y/o anticuerpos específicos de EC.

4) Analizar la evolución de la dermatosis en función de la dieta libre en gluten (DLG).

5) Analizar la evolución de la dermatosis con el tratamiento farmacológico.

\section{Materiales y métodos}

Se realizó un estudio descriptivo, observacional, retrospectivo y longitudinal de una serie de casos.

Criterios de inclusión: pacientes asistidos en servicios de dermatología y gastroenterología del Hospital de Clínicas, con diagnóstico de $\mathrm{DH}$ confirmado con histopatología y/o inmunofluorescencia directa; desde julio de 2000 a junio de 2018 inclusive. Quedaron excluidos aquellos pacientes en los que no se puedo acceder al estudio histopatológico de piel.

Se revisaron todas las historias clínicas buscando las siguientes variables:

1) Edad al diagnóstico de DH.

2) Sexo.

3) Síntomas y signos cutáneos al diagnóstico y en la evolución.

4) Prurito: definido como leve, moderado y severo, según registro en las historias clínicas.

5) Actividad de la $\mathrm{DH}$, definida como presencia de prurito y lesiones primarias.

6) Síntomas digestivos al diagnóstico: diarrea, distensión abdominal, dolor abdominal.

7) Síntomas extradigestivos al diagnóstico: anemia ferropénica.

8) Tiempo transcurrido entre el inicio de los síntomas cutáneos y el diagnóstico de $\mathrm{DH}$.

9) Presencia de EC definida como la presencia de atrofia vellositaria (MARSH II y III) y al menos un anticuerpo específico positivo.

10) Relación temporal entre el diagnóstico de $\mathrm{DH}$ y el de EC.

11) Anticuerpos específicos de EC al diagnóstico.

12) Asociación lesional con otras enfermedades autoinmunes: tiroiditis de Hashimoto, diabetes mellitus tipo 1 y vitíligo.

13) Histopatología e inmunofluorescencia directa en biopsias cutáneas.

14)Cumplimiento de la DLG, medida según registro en la historia clínica.

15)Si recibió tratamiento farmacológico (dapsona).

16) Resolución de la dermatosis a los 3 meses, 6 meses y al año.

17) Antecedentes familiares de ler grado de EC y/o $\mathrm{DH}$.

Se solicitó aprobación por parte del Comité de Ética del hospital. Se garantizó la confidencialidad de los datos obtenidos a partir de los registros clínicos, para evitar la vulneración de los derechos de los pacientes. Se presentó un consentimiento informado a los pacientes. Dado que es un estudio retrospectivo, citamos telefónicamente a aquellos pacientes que no concurrieron más a los servicios de dermatología y gastroenterología del Hospital de Clínicas. La difusión de los resultados 
obtenidos se hizo respetando las normas éticas internacionales y nacionales, y los principios éticos.

\section{Resultados}

\section{Pacientes}

Durante el período que va julio de 2000 a junio de 2018 se identificaron un total de 20 pacientes.

Se excluyeron del estudio dos pacientes: uno en el cual el diagnóstico de DH y EC se realizó en el exterior, y no contábamos con la histopatología ni IFD de la biopsia de piel y otro paciente con diagnóstico de EC confirmado, pero sin confirmación del diagnóstico de DH.

Se tomaron en cuenta solamente para el análisis de sexo, edad y los hallazgos histopatológicos e IFD, tres pacientes que tenían únicamente el diagnóstico histopatológico realizado en nuestro servicio, sin controles en nuestra policlínica ni en el servicio de gastroenterología.

Por lo tanto se incluyeron un total de 18 pacientes, 15 con DH confirmada por HP y/o IFD que se asistían en los servicios de dermatología y gastroenterología del HC. De estos, 11 eran de sexo masculino y 7 de sexo femenino. La edad media al diagnóstico de $\mathrm{DH}$ fue de 50,5 años, con una edad mínima de 22 y máxima de 72 .

\section{Dermatitis herpetiforme}

Síntomas y signos cutáneos al diagnóstico: fueron 15 los pacientes en que se pudieron analizar estos datos. El $100 \%$ de los pacientes presentó prurito severo al diagnóstico.

Con respecto a la localización de las lesiones, los 15 pacientes presentaron lesiones a nivel de codos y rodillas, 11 en glúteos, cuatro en muslos, tres en pliegue interglúteo, dorso alto y abdomen. Dos en sacro, región lumbar y brazos. Un paciente presentó lesiones en cuero cabelludo, uno en cara y en cuello, uno en dorso de pies y uno en cara palmar de dedos.

En cuanto al tipo de lesiones primarias presentadas fueron: vesículas en 9 pacientes, pápulas eritematosas en nueve pacientes, tres pacientes con ampollas, uno con lesiones prurigoides y uno con pústulas. En cuanto a las lesiones secundarias, 12 pacientes presentaron excoriaciones por rascado, ocho erosiones, tres costra hemática y dos máculas hiperpigmentadas de aspecto postinflamatorio.

El tiempo transcurrido entre el inicio de los síntomas cutáneos y el diagnóstico de $\mathrm{DH}$ fue de 0 a 36 meses, con una media de 4 meses. Cuatro pacientes tuvieron un diagnóstico inicial de escabiosis y recibieron tratamiento con ivermectina.

Para analizar los hallazgos en los estudios histopatológicos de biopsias cutáneas encontramos un total de
18 pacientes. En 12 la histopatología fue característica de $\mathrm{DH}$, mostrando decolamiento subepidérmico con formación de vesículas o ampollas y acúmulos de neutrófilos formando microabscesos a nivel del vértice de las papilas dérmicas. En tres pacientes la histopatología mostró una dermatitis espongiótica, en uno pigmentación postinflamatoria y en dos pacientes no se observan alteraciones. Tres pacientes tuvieron leucocitoclasia. Dos pacientes tenían una biopsia previa, las cuales habían informado prurigo y eccema.

Se realizó estudio de IFD a 12 pacientes de un total de 18. Los seis pacientes a los que no se les realizó la IFD presentaban una histopatología característica de DH. 11 presentaron depósito granular de IgA a nivel papilar, y tres presentaban además el depósito de IgA a lo largo de la unión dermoepidérmica. En 11, los depósitos fueron de IgA, cinco presentaban además depósitos de $\mathrm{C} 3$, y 4 presentaban depósitos concomitantes de IgG. Un paciente con HP característica de DH mostró en la IFD cuerpos coloides con IgM únicamente.

Evolución de la DH a 3 meses del diagnóstico: tenemos el dato en nueve pacientes, todos mantuvieron actividad de la enfermedad, tres agregaron máculas hiperpigmentadas de aspecto postinflamatorio.

Evolución de la DH a 6 meses del diagnóstico: cuatro pacientes refirieron cumplir DLG, tres con la enfermedad en actividad, un paciente sin prurito y sin lesiones primarias. Contamos con la evolución de la DH a 12 meses del diagnóstico en cinco pacientes. Entre ellos están los cuatro pacientes que recibieron tratamiento con dapsona. Tres de ellos se presentaron al año sin actividad de la DH. El restante mantuvo actividad de la $\mathrm{DH}$ bajo dapsona, presentando pápulas eritematosas y prurito moderado.

Un paciente con control al año sin tratamiento con dapsona presentó buena adherencia a la DLG, sin actividad de la enfermedad. Más allá del año de evolución tenemos control en dos pacientes que habían abandonado en forma intermitente la dapsona y la DLG, reapareciendo las lesiones activas de DH. Cuatro pacientes recibieron dapsona, con una dosis entre 50-100 mg día. En dos pacientes se registró un descenso de la hemoglobina que requirió disminución de la dosis. No se registraron efectos adversos graves.

\section{Enfermedad celíaca}

En 13 de 15 pacientes la DH fue el síntoma guía para el diagnóstico de EC. En siete pacientes se indicó la DLG desde el servicio de dermatología.

En cuanto a los síntomas y signos de EC al diagnóstico, se presentaron cinco pacientes asintomáticos, seis pacientes con síntomas clásicos de EC y cuatro pacientes con presentación no clásica, dos de estos últimos presentaron únicamente anemia ferropénica como 
manifestación extradigestiva, sin síntomas digestivos. Cinco pacientes presentaron diarrea, cinco distensión abdominal, cuatro dolor abdominal. Cinco con anemia $\mathrm{y}$ dos con hipocrecimiento.

De las seis pacientes de sexo femenino, cuatro tuvieron abortos espontáneos, dos en número mayor o igual a 2. Dos con hijos prematuros.

Anticuerpos de EC al diagnóstico: tenemos esa información en 14 de los 15 pacientes. Los anticuerpos antitransglutaminasa resultaron positivos en 12 pacientes, los dos restantes con resultado negativo se encontraban bajo DLG al tomar la muestra. En 12 pacientes se midieron los anticuerpos antiendomisio al diagnóstico, resultando positivos en 10 pacientes y negativos en los dos pacientes bajo DLG. Se midieron los anticuerpos antigliadina en 13 pacientes, fueron positivos en ocho y negativos en cinco pacientes, dentro de los cuales estaban aquellos bajo DLG. Cinco pacientes presentaron anemia ferropénica al diagnóstico.

Resultados de fibrogastroscopía e histopatología de biopsias intestinales: estudio endoscópico: un paciente no se realizó el estudio endoscópico; de los 14 restantes tres no tenían alteraciones mucosas en la HP (Marsh 0), uno Marsh I, uno Marsh II, cinco Marsh IIIA, tres IIIB y uno IIIC.

De los tres pacientes con Marsh 0, dos presentaron anticuerpos de EC positivos y el restante anticuerpos negativos. En este último paciente la biopsia y los anticuerpos se realizaron bajo DLG. Los pacientes con Marsh I y II tenían anticuerpos positivos. Dos pacientes tenían el antecedente familiar de EC en familiares de primer grado.

Sólo en tres de los 15 pacientes se estudiaron los familiares de primer grado para EC, en un caso se observaron hermanos positivos asintomáticos.

Adherencia a DLG: seis pacientes lograron adherencia sostenida a DLG, medida por la respuesta del paciente sobre esta en los controles.

\section{Asociación lesional}

Cuatro pacientes con hipotiroidismo, no contábamos con anticuerpos antitiroideos por lo cual no pudimos definir si era debido a enfermedad de Hashimoto. No había pacientes con diabetes mellitus tipo 1 ni vitíligo.

\section{Discusión}

\section{Características demográficas}

En cuanto a la distribución por sexo tenemos 11 pacientes de sexo masculino y 7 de sexo femenino, constituyendo un radio hombre mujer de 1,5:1, igual a lo reportado en la literatura ${ }^{(1,7)}$.

La edad media al diagnóstico en nuestra muestra fue de 50,5 años, mayor a la reportada en la literatura que es de $42,8^{(1,7,11)}$. Podría explicar esto que nuestra muestra no incluye niños, ya que en el Hospital de Clínicas se atienden pacientes de 15 años o más; y también el pequeño tamaño de la muestra.

\section{Dermatitis herpetiforme}

Con respecto a la presentación clínica de la dermatosis la localización y morfología de las lesiones fue característica. Todos los pacientes presentaron lesiones simétricas en áreas extensoras de miembros, y en orden decreciente en glúteos, muslos, dorso, hipogastrio, pliegue interglúteo, región lumbosacra, brazos, cuello, cuero cabelludo, dorso de pies y cara palmar de dedos de la mano. Como presentación atípica un paciente presentó lesiones en cara, pero acompañadas de lesiones de localización clásica, a diferencia de los casos reportados con compromiso facial aislado ${ }^{(14)}$.

Las lesiones fueron polimorfas, con pápulas eritematosas y vesículas agrupadas sobre una base eritematosa como lesiones primarias; y como lesiones secundarias erosiones y excoriaciones secundarias al rascado y máculas hiperpigmentadas postinflamatorias. No tuvimos presentaciones urticariformes ni púrpura palmoplantar en nuestra muestra.

Si bien la presentación clínica fue clásica de $\mathrm{DH}$, no se realizó rápidamente el diagnóstico, con tiempos entre el inicio de los síntomas y el diagnóstico de hasta 36 meses, y con tratamientos con planteo de escabiosis en cuatro de los 15 pacientes. Podría influir aquí la demora en el acceso a la consulta dermatológica.

El estudio histopatológico fue característico de $\mathrm{DH}$ en 12 de los 18 pacientes estudiados. En seis pacientes los hallazgos no fueron característicos, pudiendo tratarse de lesiones más evolucionadas ${ }^{(15,16)}$.

Se realizó estudio de IFD en 12 pacientes porque se perdieron muestras para IFD por errores como colocación en formol en lugar de suero fisiológico o no refrigeración de la misma. De todas formas, estos seis pacientes a los que no se les realizó IFD presentaban una histopatología característica para DH. Once presentaron depósito granular de IgA a nivel papilar, y tres presentaron además el depósito de IgA a lo largo de la unión dermoepidérmica, hallazgos diagnósticos de la IFD ${ }^{(15)}$. Ningún paciente de nuestra muestra presentó un patrón fibrilar de IgA, dato que no llama la atención ya que está descrito sólo en un $1,2 \%$ a $2,6 \%$ de la población occidental ${ }^{(17)}$. Además de los depósitos de IgA, presentaron en orden descendente depósitos concomitantes de $\mathrm{C} 3$ (cinco pacientes), IgG (cuatro pacientes) y un paciente depósitos de IgM. Los depósitos de IgM son los que siguen en frecuencia a los de IgA en la literatura, a diferencia de nuestra muestra ${ }^{(17)}$.

En cuanto a la evolución de la dermatosis, los datos son más débiles por la pérdida de pacientes en contro- 
les sucesivos. No tenemos datos específicos en todos los pacientes sobre el motivo de la baja adherencia a los controles. Podría ser la dificultad económica para concurrir a los controles, muchas veces presente en los pacientes que se asisten en el servicio público de salud. Un paciente se cambió a la asistencia mutual luego del diagnóstico.

Como ya se mencionó, las lesiones cutáneas demoran 1-2 años en lograr su remisión completa con la DLG, y reaparecen rápidamente luego de la reintroducción del gluten en la dieta ${ }^{(5)}$. A los tres meses del diagnóstico todos los pacientes ${ }^{(7)}$ mantenían prurito y lesiones activas. A los seis meses del diagnóstico tres de cuatro pacientes mantenían lesiones activas. Sólo contamos con la evolución de la DH a 12 meses del diagnóstico en cinco pacientes. Sin lesiones activas de la dermatosis, pero cuatro de ellos bajo tratamiento farmacológico con dapsona. Más allá del año de evolución, tuvimos control en dos pacientes que habían abandonado en forma intermitente la dapsona y la DLG, reapareciendo las lesiones activas de $\mathrm{DH}$.

La dapsona no está disponible en Uruguay, los pacientes que han recibido tratamiento con este fármaco lo compran en el exterior (Argentina). Esto dificulta la constancia del tratamiento, con interrupciones transitorias por no acceder a la medicación. Tres de cuatro pacientes que la habían recibido mostraron la rápida respuesta de la dermatosis referida en la literatura ${ }^{(5,18)}$. En cuanto a los efectos adversos, dos pacientes presentaron descenso de la $\mathrm{Hb}$ que requirió disminución de la dosis. Ninguno presentó metahemoglobinemia ni efectos idiosincráticos.

\section{Enfermedad celíaca}

\section{Síntomas y signos de EC al diagnóstico}

Se observaron sólo cinco pacientes asintomáticos $(33 \%)$. Este resultado discrepa con lo descrito en la literatura, donde un $60 \%-70 \%$ de los pacientes con $\mathrm{DH}$ tienen una EC asintomática ${ }^{(19,20)}$. Esta diferencia podría explicarse por el bajo número de pacientes en nuestro estudio. Otra explicación sería que los pacientes con EC de un nivel socioeconómico bajo tienen más posibilidades de presentarse con diarrea u otros síntomas gastrointestinales en comparación con los de mayor ingreso $^{(21,22)}$, y la población del Hospital de Clínicas que es un centro de asistencia público podría presentarse de esta forma.

En cuanto a los anticuerpos antitransglutaminasa y antiendomisio al diagnóstico, estos fueron negativos en los pacientes que se estudiaron bajo DLG. Como ya mencionamos, estos son marcadores de la adherencia a la DLG con la que se negativizan, y su medición para el diagnóstico se debe realizar consumiendo gluten ${ }^{(4,10)}$.

Resultados de la histopatología de biopsias intesti- nales: $9 / 14$ pacientes de nuestro estudio $(64,2 \%)$ presentaron atrofia vellositaria e hiperplasia de las criptas (Marsh III), similar al 70\% reportado en la literatura. 4/14 (28,5\%) con atrofia vellositaria severa (Marsh IIIb y IIIc), lo que coincide con el $29 \%$ reportado $^{(1,8,23)}$.

De los tres pacientes con Marsh 0, dos presentaron anticuerpos de EC positivos y el restante anticuerpos negativos. En este último paciente la biopsia y los anticuerpos se realizaron bajo DLG y no teníamos controles posteriores. Los pacientes con Marsh I y II tenían anticuerpos positivos.

Por lo que confirmamos el diagnóstico de EC mediante la presencia de atrofia vellositaria y/o anticuerpos específicos en 14 de los 15 pacientes.

Seis pacientes $(40 \%)$ adhirieron a la DLG. Los niveles reportados de adherencia estricta a la DLG en adultos con EC variaron entre 36\%-96\%(24). Si bien la DLG ofrece múltiples beneficios en el manejo de la $\mathrm{DH}$, en la práctica no es bien aceptada por muchos pacientes. Requiere una monitorización detallada de todos los alimentos ingeridos, consume tiempo y restringe socialmente. Además, los productos libres de gluten no están ampliamente disponibles y son más costosos que su contraparte con gluten ${ }^{(25)}$. Puede ocurrir una ingesta inadvertida de gluten por contaminación cruzada, llevando a que los pacientes mantengan lesiones cutáneas y lesiones en la mucosa intestinal inducidas por gluten. Medicamentos y suplementos pueden presentar trazas de gluten en sus excipientes ${ }^{(3)}$.

Los factores que se asocian significativamente con una mejor adherencia a la DLG son la comprensión de la dieta, la capacidad de mantenerla cuando se ingieren alimentos fuera del hogar y la participación en grupos de apoyo ${ }^{(24,26)}$. En Uruguay contamos con la asociación de celíacos del Uruguay (ACELU), a la que se vinculan los pacientes desde el servicio de gastroenterología. La adherencia a la DLG minimiza los riesgos asociados a la exposición prolongada al gluten en pacientes con DH y EC ${ }^{(25)}$.

\section{Asociación lesional}

En trabajos no recientes se reportan $4,3 \%$ de pacientes con tiroiditis de Hashimoto asociada a $\mathrm{DH}^{(27)}$. Para EC en general es la enfermedad autoinmune más comúnmente asociada, presente en $20 \%-30 \%$ de los pacientes ${ }^{(13)}$. $4 / 15$ pacientes del estudio $(26,6 \%)$ tenían hipotiroidismo asociado, no pudimos definir si este se debía a tiroiditis de Hashimoto ya que no contábamos con la determinación de anticuerpos antitiroideos, pero la incidencia coincide con lo reportado para EC.

\section{Conclusiones}

En 13 de 15 pacientes la DH fue el síntoma guía para el diagnóstico de EC. 
Se destaca la importancia para el médico dermatólogo de reconocer la $\mathrm{DH}$, ya que de permanecer sin diagnóstico los pacientes persisten con síntomas cutáneos intermitentes que afectan su calidad de vida; y están expuestos al desarrollo de complicaciones de la enteropatía subyacente y aumento del riesgo de linfoma.

No debemos iniciar DLG hasta confirmación del diagnóstico de EC, ya que con ésta negativizan los anticuerpos y mejora a la enteropatía, pudiendo dificultar la confirmación diagnóstica de EC.

Es muy importante el trabajo interdisciplinario en estos pacientes, en conjunto con gastroenterólogo y nutricionista. Es fundamental lograr una adherencia a la DLG, que lleva a la resolución de la dermatosis y permite disminuir o suspender el tratamiento con dapsona y los riesgos asociados a este; y en la enteropatía mejoría de los síntomas digestivos y extradigestivos. La DLG mejora la calidad de vida de los pacientes y reduce el riesgo de desarrollo de linfoma.

\section{Agradecimientos}

Prof. Adj. Dra. María Eugenia Mazzei, por su ayuda y disposición, para la revisión de biopsias de las que se había extraviado el registro, y a la Profesora Adjunto de la Cátedra de Gastroenterología Carolina Olano.

\section{Summary}

Introduction: dermatitis herpetiformis (DH) is an inflammatory cutaneous disease comprising relapses and remissions. It is an extraintestinal manifestation of coeliac disease, which can even be its first clinical manifestation. Coeliac disease (CD) is an autoimmune systemic disease, associated with enteropathy, that is induced by the intake of gluten in genetically predisposed individuals. In both diseases an association between HLA DQ2 and HLA DQ8 has been proved.

Objective: to present the characteristics of patients with Dermatitis herpetiformis (DH) and Coeliac disease (CD) who are assisted in the dermatology and gastroenterology polyclinics at the "Dr. Manuel Quintela" University Hospital.

Methodology: descriptive, observational, retrospective and longitudinal study of a series of cases. Inclusion criteria: patients assisted at the dermatology and gastroenterology services of the University Hospital, with a diagnosis of Dermatitis herpetiformis (DH) confirmed by histopathology and/or direct immunofluorescence (DIF), from July, 200 to June 2018 inclusive. Patients whose skin histopathological exam could not be accessed were excluded from the study.

Results: 15 patients were included, 9 male and 6 female. Average age upon diagnosis of $\mathrm{DH}$ is 49 years old. 4 patients were treated with dapsona, evidencing a fast response to dermatosis, and no severe adverse effects. In 13 patients, DH was the guiding symptom to diagnose coeliac disease. However, 5 patients had asymptomatic $\mathrm{CD}, 6$ presented classical symptoms and 4 non-classical symptoms.

\section{Resumo}

Introdução: a dermatite herpetiforme (DH) é uma doença inflamatória da pele que ocorre em impulsos e remissões. É uma manifestação extra-intestinal da doença celíaca (DC), da qual pode ser a primeira manifestação clínica. A doença celíaca é uma doença autoimune sistêmica associada à enteropatia, desencadeada pela ingestão de glúten em indivíduos geneticamente predispostos. Uma associação com HLA DQ2 e HLA DQ8 foi demonstrado em ambas as doenças.

Objetivo: mostrar as características dos pacientes com HD e DC atendidos nas policlínicas dermatológicas e gastroenterológicas do "Dr. Manuel Quintela".

Metodologia: foi realizado um estudo descritivo, observacional, retrospectivo e longitudinal de uma série de casos. Critérios de inclusão: pacientes atendidos nos serviços de dermatologia e gastroenterologia do Hospital de Clínicas, com diagnóstico de HD confirmado pela histopatologia e / ou imunofluorescência direta; de julho de 2000 a junho de 2018 inclusive. Foram excluídos os pacientes em que não foi possível acessar o estudo histopatológico da pele.

Resultados: incluímos um $\mathrm{N}$ total de 15 pacientes, 9 homens e 6 mulheres. A idade média no diagnóstico de DH é de 49 anos. 4 pacientes receberam tratamento com dapsona, com resposta rápida da dermatose, sem efeitos adversos graves. Em 13 pacientes, a DH foi o sintoma norteador para o diagnóstico de DC. 5 pacientes com DC assintomática, 6 com sintomas clássicos, 4 com sintomas não clássicos.

\section{Bibliografía}

1. Collin P, Salmi T, Hervonen K, Kaukinen K, Reunala T. Dermatitis herpetiformis: a cutaneous manifestation of coeliac disease. Ann Med 2017; 49(1):23-31. doi: 10.1080/07853890.2016.1222450.

2. Rodrigo L, Beteta-Gorriti V, Álvarez N, Gómez de Castro C, de Dios A, Palacios L, et al. Cutaneous and mucosal manifestations associated with celiac disease. Nutrients 2018; 10(7):800. doi: 10.3390/nu10070800.

3. Lebwohl B, Sanders D, Green P. Coeliac disease. Lancet 2018; 391(10115):70-81. doi: 10.1016/S01406736(17)31796-8.

4. Argentina. Ministerio de Salud. Dirección Nacional de Promoción de la Salud y Control de Enfermedades Crónicas No Transmisibles. Documento de Consenso de Enfermedad Celíaca 2017. Buenos Aires: Ministerio de Salud, 2017. Disponible en: http://iah.salud.gob.ar/doc/Documento232.pdf [Consulta: 24 marzo 2021].

5. Caproni M, Antiga E, Melani L, Fabbri P. Guidelines 
for the diagnosis and treatment of dermatitis herpetiformis. J Eur Acad Dermatol Venereol 2009; 23(6):633-8. doi: 10.1111/j.1468-3083.2009.03188.x.

6. Clarindo M, Possebon A, Soligo E, Uyeda H, Ruaro R, Empinotti J. Dermatitis herpetiformis: pathophysiology, clinical presentation, diagnosis and treatment. An Bras Dermatol 2014; 89(6):865-75. doi: 10.1590/abd18064841.20142966.

7. Bolotin D, Petronic-Rosic V. Dermatitis herpetiformis. Part I: epidemiology, pathogenesis, and clinical presentation. J Am Acad Dermatol 2011; 64(6):1017-24. doi: 10.1016/j. jaad.2010.09.777.

8. Reunala T. Dermatitis herpetiformis. Clin Dermatol 2001; 19(6):728-36. doi: 10.1016/s0738-081x(00)00184-x.

9. Bonciolini V, Bonciani D, Verdelli A, D'Errico A, Antiga E, Fabbri P, et al. Newly described clinical and immunopathological feature of dermatitis herpetiformis. Clin Dev Immunol 2012; 2012:967974. doi: 10.1155/2012/967974.

10. Keuning J, Peña A, van Leeuwen A, van Hooff J, va Rood J. HLA-DW3 associated with coeliac disease. Lancet 1976; 1(7958):506-8. doi: 10.1016/s0140-6736(76)90294-4.

11. Salmi T, Hervonen K, Kautiainen H, Collin P, Reunala T. Prevalence and incidence of dermatitis herpetiformis: a 40-year prospective study from Finland. Br J Dermatol 2011; 165(2):354-9. doi: 10.1111/j.1365-2133.2011.10385.x.

12. Durán S, Ciriacos C, coord. Guía de detección precoz, diagnóstico y tratamiento de la enfermedad Celíaca. Montevideo: MSP, 2017. Disponible en: https://www.gub.uy/ministerio-salud-publica/comunicacion/publicaciones/guia-de-deteccion-precoz-diagnostico-y-tratamiento-de-la-enfermedad [Consulta: 24 marzo 2021].

13. Hujoel I, Reilly N, Rubio-Tapia A. Celiac disease: clinical features and diagnosis. Gastroenterol Clin North Am 2019; 48(1):19-37. doi: 10.1016/j.gtc.2018.09.001.

14. Cinats A, Parsons L, Haber R. Facial Involvement in Dermatitis Herpetiformis: A Case Report and Review of the Literature. J Cutan Med Surg 2019; 23(1):35-7. doi: $10.1177 / 1203475418795818$.

15. Weedon D, Strutton G, Rubin A. Weedon's skin pathology. 3. ed. London: Churchill-Livingstone-Elsevier, 2010.

16. Warren S, Cockerell C. Characterization of a subgroup of patients with dermatitis herpetiformis with nonclassical his- tologic features. Am J Dermatopathol 2002; 24(4):305-8. doi: 10.1097/00000372-200208000-00003.

17. Barnadas M. Dermatitis herpetiformis: a review of direct immunofluorescence findings. Am J Dermatopathol 2016; 38(4):283-8. doi: 10.1097/DAD.0000000000000420.

18. Herrero-González J. Guía clínica de diagnóstico y tratamiento de la dermatitis herpetiforme. Actas Dermosifiliogr 2010; 101(10):820-6.

19. Kárpáti S. Dermatitis herpetiformis: close to unravelling a disease. J Dermatol Sci 2004; 34(2):83-90. doi: 10.1016/j. jdermsci.2003.11.004.

20. Zone J. Skin manifestations of celiac disease. Gastroenterology 2005; 128(4 Suppl 1):S87-91. doi: 10.1053/j.gastro.2005.02.026.

21. Roy A, Mehra S, Kelly C, Tariq S, Pallav K, Dennis M, et al. The association between socioeconomic status and the symptoms at diagnosis of celiac disease: a retrospective cohort study. Therap Adv Gastroenterol 2016; 9(4):495-502. doi: 10.1177/1756283X16637532.

22. Olén O, Bihagen E, Rasmussen F, Ludvigsson J. Socioeconomic position and education in patients with coeliac disease. Dig Liver Dis 2012; 44(6):471-6. doi: 10.1016/j. dld.2012.01.006.

23. Mansikka E, Hervonen K, Salmi T, Kautiainen H, Kaukinen K, Collin P, et al. The decreasing prevalence of severe villous atrophy in dermatitis herpetiformis: a 45 -year experience in 393 patients. J Clin Gastroenterol 2017; 51(3):235-9. doi: 10.1097/MCG.0000000000000533.

24. Hall N, Rubin G, Charnock A. Systematic review: adherence to a gluten-free diet in adult patients with coeliac disease. Aliment Pharmacol Ther 2009; 30(4):315-30. doi: 10.1111/j.1365-2036.2009.04053.x.

25. Antiga E, Caproni M. The diagnosis and treatment of dermatitis herpetiformis. Clin Cosmet Investig Dermatol 2015; 8:257-65. doi: 10.2147/CCID.S69127.

26. Leffler D, Edwards-George J, Dennis M, Schuppan D, Cook F, Franko D, et al. Factors that influence adherence to a gluten-free diet in adults with celiac disease. Dig Dis Sci 2008; 53(6):1573-81. doi: 10.1007/s10620-007-0055-3.

27. Reunala T, Collin P. Diseases associated with dermatitis herpetiformis. Br J Dermatol 1997; 136(3):315-8.

\footnotetext{
Contribución de autores versión. versión.

Mariana Lizarraga ORCID 0000-0002-8446-1976

Pilar Echenagusía ORCID 0000-0002-79267263

Julio Magliano ORCID 0000-0003-2007-9871

Carlos Bazzano ORCID 0000-0003-1960-3365

Miguel Martínez Asuaga ORCID 0000-0002-5729-0702
}

Mariana Lizarraga, Pilar Echenagusia, Julio Magliano participaron en la concepción, diseño, adquisición de datos, análisis, interpretación de resultados, escritura del manuscrito, aprobación de última versión.

Carlos Bazzano participó en concepción, diseño, adquisición de datos, análisis, interpretación de resultados, aprobación de última

Miguel Martínez Asuaga participó en la concepción, adquisición de datos, interpretación de resultados, aprobación de última 